\title{
Investigational agents in the treatment of Parkinson's disease: focus on safinamide
}

This article was published in the following Dove Press journal:

Journal of Experimental Pharmacology

14 August 2012

Number of times this article has been viewed

\section{Naveed M Malek \\ Donald G Grosset}

Department of Neurology, Institute of Neurological Sciences, Southern General Hospital, Glasgow, UK
Correspondence: Naveed Malek Ward 67, Southern General Hospital, Glasgow G5I 4TF, UK

Tel +44 I4I 20I 2486

Email nmalek@nhs.net
Abstract: The authors review management issues in Parkinson's disease (PD) and provide an overview of the current pharmacological management strategies, with a specific focus on safinamide. Current therapeutic management of PD largely involves strategies to optimize the replacement of deficient dopamine, using levodopa, dopamine agonists, and inhibitors of dopamine-metabolizing enzymes. Currently under investigation for use in the treatment of PD, safinamide has multiple modes of action including monoamine oxidase B inhibition. It is well absorbed orally, has a long plasma half-life, and does not have liver enzyme-inducing or liver enzyme-inhibiting activity. Peak plasma concentration occurs 2-4 hours after single oral doses. Safinamide as monotherapy and as an adjunct to dopamine agonists improves Unified Parkinson's Disease Rating Scale motor scores. One randomized, placebo-controlled trial involving 168 patients given a median safinamide dose of $70 \mathrm{mg}$ /day (range 40-90 mg/day) significantly increased the proportion of responders - defined as patients improving their Unified Parkinson's Disease Rating Scale motor scores by $30 \%$ or more from baseline - after 3 months $(37.5 \%$ for safinamide versus $21.4 \%$ for placebo; $P<0.05$ ). Safinamide increased "on" time with no or minor dyskinesia compared with the placebo in another trial, but dyskinesia severity was not reduced. Safinamide was well tolerated, with an adverse effect profile similar to that of the placebo. Further Phase III trial data for safinamide efficacy is awaited, and will be of interest in a comparison with other developments in PD therapeutics: modified formulations of available compounds, new drug classes such as adenosine receptor antagonists, and gene-based therapies.

Keywords: monoamine oxidase B inhibitors, dyskinesia, Unified Parkinson's Disease Rating Scale, antiparkinsonian drug

\section{Introduction to the management issues in Parkinson's disease}

Parkinson's disease (PD) is a chronic neurodegenerative disorder characterized by a deficiency of dopamine in the nigrostriatal pathway. It is recognized by the core motor symptoms of bradykinesia, rigidity, tremor, and postural instability that help to make the clinical diagnosis of PD (according, for example, to the UK Parkinson's Disease Society Brain Bank clinical diagnostic criteria ${ }^{1}$ ), but nonmotor symptoms such as sleep disturbances, olfactory dysfunction, and behavioral and cognitive problems can also cause considerable functional disability. Management initially involves a discussion of the diagnosis and nature of the condition with the patient, followed by a combined approach to ameliorate 
the motor and nonmotor symptoms, with pharmacotherapy and physical, occupational, and speech therapy.

\section{Overview of current pharmacological management strategies}

Since the discovery of levodopa as a treatment for PD, additional treatments, with fewer long-term side effects such as dyskinesia and with a better pharmacokinetic profile than the short half-life of levodopa (1-2 hours), have been developed. ${ }^{2}$ Over the last decade this search has been further motivated by discoveries in the understanding of the pathological mechanisms underlying PD. Available PD treatment options largely improve motor symptoms rather than being neuroprotective, although there is an indication that some agents may fulfill both objectives. ${ }^{3}$ Current pharmacological management of the motor symptoms of PD relies mainly on dopamine precursors (levodopa), dopamine agonists (DAs), enzyme inhibitors of monoamine oxidase B (MAO-B) and catechol-O-methyltransferase (COMT), and amantadine, which is an N-methyl-D-aspartate antagonist.

Levodopa was first introduced in the 1960s for the treatment of PD and remains the gold standard. It is an amino acid precursor of dopamine and acts by replenishing striatal dopamine. Levodopa is combined with an extracerebral dopa decarboxylase inhibitor (DDI), either benserazide or carbidopa, to inhibit peripheral breakdown and enhance central delivery. Levodopa-based treatment may cause nausea, vomiting, and postural hypotension in the early stages, and it is linked to the development of dyskinesia in the later stages when response fluctuations emerge. It causes fewer neuropsychiatric side effects than other antiparkinsonian drugs and is, therefore, a common first choice for older patients and those with comorbidities.

DAs bind directly to the postsynaptic dopamine receptors and are effective in improving function in patients with PD, both as monotherapy and as adjuncts to levodopa. The ergot-derived DAs (bromocriptine, cabergoline, and pergolide) have fallen out of favor because of their fibrogenic potential. Non-ergot DAs including pramipexole, ropinirole, and rotigotine are often first-line agents in younger-onset PD patients, where the risk of longer-term motor complications is higher. Side effects include nausea, hypotension, peripheral edema, tiredness, and impulse control disorders.

Monoamine oxidase (MAO) is one of the enzymes that catalyze the breakdown of dopamine. Selective MAO-B inhibitors selegiline and rasagiline are effective as monotherapy and as adjuncts to levodopa or other agents, without having the dietary restrictions of nonselective MAO inhibitors. When used with levodopa, selegiline may accentuate postural hypotension. Rasagiline is contraindicated in severe hepatic impairment.

COMT is another enzyme that catabolizes dopamine, both peripherally and in the central nervous system. Inhibition of COMT in the periphery allows for increased bioavailability and longer duration of action of levodopa. Entacapone and tolcapone are used as adjuncts to levodopa for PD patients who experience "end of dose" deterioration. Because of the hepatotoxic potential of tolcapone, entacapone is the preferred COMT inhibitor. Entacapone is contraindicated in those with pheochromocytoma, a history of neuroleptic malignant syndrome, or nontraumatic rhabdomyolysis. Side effects include diarrhea, nausea, dry mouth, and sweating. It is available as a triple combination tablet with levodopa and a DDI, marketed as Stalevo ${ }^{\circledR}$ (Novartis International AG, Basel, Switzerland).

Amantadine is an N-methyl-D-aspartate antagonist and a weak DA with modest antiparkinsonian effects. It is used as an antidyskinetic agent in PD. It is contraindicated in epilepsy and in those with a history of gastric ulceration. Tolerance to its effects may develop, and confusion and hallucinations occasionally occur.

Anticholinergic drugs (orphenadrine, procyclidine, and trihexyphenidyl) exert their antiparkinsonian action by reducing the effects of relative central cholinergic excess that occurs as result of dopamine deficiency. However, the use of anticholinergic drugs has declined significantly because of adverse cognitive effects; other side effects include dry mouth, constipation, and urinary retention.

In the early stages of the disease, DAs, MAO-B inhibitors, and levodopa with a DDI are the main choices for the symptomatic treatment of the motor symptoms of PD. There is no single drug of choice. Physician and patient preference, considering the adverse effect profile of individual drug classes, and comorbidities influence the choice and sequence of drug therapy. Current approaches favoring DAs initially for many young-onset patients who are otherwise well and levodopa as the first choice for older patients (often with comorbidity) may change with results of the Parkinson's Disease Medicines (PDMED) trial, although full results are not yet reported. ${ }^{4}$ The management of later stages of PD often involves combinations of dopamine replacement therapies, such as levodopa with a DDI, DAs, MAO-B inhibitors, and COMT inhibitors. However, motor and nonmotor complications may limit drug choices and require individualization of drug therapy. Parenteral routes of drug delivery (apomorphine, levodopa plus carbidopa intestinal gel) are utilized when motor complications become more troublesome, providing a more physiologic nonpulsatile delivery of dopamine. 


\section{Safinamide}

MAO plays a major role in the in vivo inactivation of biogenic and diet-derived amines, in both the central nervous system and the peripheral body tissues. MAO isoenzyme A (MAO-A) is mainly responsible for the deamination of serotonin and noradrenaline in the intestine. The MAO-B isoenzyme predominates in the striatum. ${ }^{5}$

Dopamine is a substrate for both MAO-A and MAO-B. Selective inhibitors of MAO-B reduce the catabolism of dopamine and thereby increase the presynaptic levels of this neurotransmitter in the basal ganglia, without affecting the metabolism of other amines that are catabolized by MAO-A.

Safinamide mesylate was first synthesized in 1989 in the medicinal chemistry program at Farmitalia Carlo Erba (Milan, Italy). Initial investigation of safinamide was for its use as a potential antiepileptic drug, following the observation that milacemide, from which safinamide is derived, had some anticonvulsant activity. ${ }^{6}$ Safinamide mesylate is an alphaaminoamide (chemical formula $\mathrm{C}_{17} \mathrm{H}_{19} \mathrm{FN}_{2} \mathrm{O}_{2} \cdot \mathrm{CH}_{4} \mathrm{O}_{3} \mathrm{~S}$ ) with multiple mechanisms of action.

\section{Mechanism of action}

Sodium channel- and calcium channel-blocking properties led to initial trials of safinamide as an antiepileptic drug, but its selective and reversible MAO-B inhibitor activity drew attention toward its potential use in PD. ${ }^{7-10}$ The selectivity of safinamide for the $\mathrm{B}$ isoform of the human enzyme versus the $\mathrm{A}$ isoform is about 1000:1, compared with selegiline at $3400: 1$ and rasagiline at 2500:1.,11,12

Safinamide also inhibits dopamine reuptake and glutamate release, the latter action being shared with amantadine, which has some efficacy in reducing dyskinesia in PD. ${ }^{13-16}$ The main safinamide mechanism of action is inhibition of the sigma-1 receptor, where the half maximal inhibitory concentration (IC50) is $19 \mathrm{nM}^{8}$ The potency of safinamide is less at other sites, being in the micromolar range for sodium channel blockade (IC50, $8.2 \mu \mathrm{M}$ ) and the high micromolar range for calcium channel blockade (IC50, $31.5 \mu \mathrm{M})$ and glutamate release (IC50, $>56.4 \mu \mathrm{M}$ ). Its potency in blocking MAO-B is in the submicromolar range (IC50, $450 \mathrm{nM}){ }^{8,17,18}$ Sigma-1 receptors are unique chaperone proteins that reside in the endoplasmic reticulum of cells. Initially considered a subtype of the opioid receptor subfamily, sigma-1 receptors are now thought to be multifunctional regulatory proteins with a role in central nervous system development, plasticity, and neurodegenerative disorders. ${ }^{19}$ Other drugs that inhibit sigma-1 receptors (eg, Anavex 2-73, Anavex Life Sciences Corporation, Vancouver, Canada) have, like safinamide, been investigated as antiepileptic drugs. The role of sigma-1 receptor inhibition in PD is unclear; this mode of action may not be relevant to safinamide's antiparkinsonian effect. ${ }^{20}$

Dopamine metabolism produces free radicals and these are believed to contribute to oxidative stress in nigral cells. In blocking the first step of dopamine metabolism by inactivating MAO-B, safinamide may reduce the production of free radicals, but this is expected to be a class effect. ${ }^{21}$

The multiple pharmacodynamic actions of safinamide make it a suitable candidate for evaluation as a neuroprotective agent against excitotoxicity and oxidative damage leading to neuronal cell death in PD.

\section{Pharmacokinetics}

Marzo et $\mathrm{al}^{22}$ administered safinamide orally to eight healthy human volunteers in the dose range of $0.025-10 \mathrm{mg} / \mathrm{kg}$ in single doses and $1.25-5 \mathrm{mg} / \mathrm{kg}$ in a repeat-dose regimen. It was well absorbed systemically, with peak plasma concentrations reached at 2-4 hours after single doses and 5-6 hours after repeat dosing. The maximum concentration at steady state was $1.5-1.7$ times higher than the maximum concentration after single dosing, suggesting no significant safinamide accumulation in subjects with normal excretory function. The clearance half-life was about 22 hours and $89 \%$ was bound to plasma proteins.

Both dose linearity and dose proportionality were demonstrated after enteral absorption. The ingestion of food heavy in fat prior to oral dosing of safinamide resulted in a more sustained rate of absorption, without affecting the extent of absorption. ${ }^{22}$ Approximately $70 \%$ of an ingested dose is metabolized to a major inactive Phase I metabolite in plasma and is conjugated to a second major Phase II metabolite in urine. ${ }^{23}$

\section{Pharmacodynamics}

In human studies, the MAO-B enzyme was partially and dose-proportionally inhibited in the dose range $25-150 \mu \mathrm{g} / \mathrm{kg}$ of oral safinamide. Safinamide plasma levels of about $200 \mathrm{ng} / \mathrm{mL}$, reached with a $600 \mu \mathrm{g} / \mathrm{kg}$ oral dose, caused 91\% MAO-B inhibition, suggesting that doses greater than $300-600 \mu \mathrm{g} / \mathrm{kg}$ at steady state would totally inhibit MAO-B. The median effective dose for MAO-B inhibition was $87.5 \mu \mathrm{g} / \mathrm{kg}{ }^{20}$

Safinamide potentiates levodopa-mediated increases in dopamine levels in dopamine-depleted mice and reverses the waning motor response after prolonged levodopa treatment in rats with 6-hydroxydopamine lesions. ${ }^{14}$ 
Safinamide has been reported to have neuroprotective effects and to be able to counteract excitotoxin-induced hippocampal neuronal death in animal models..$^{9,24}$

In the mouse model, safinamide has been reported to fully prevent forebrain dopamine depletion and neuronal cell death in the substantia nigra if given prior to 1-methyl-4phenyl-1,2,3,6-tetrahydropyridine (MPTP), a toxin known to produce parkinsonism. This preventive effect is shared with the other MAO-B inhibitors selegiline and rasagiline. ${ }^{24,25}$

\section{Safety and tolerability}

Safinamide was well tolerated in human studies. Safinamide was not found to be genotoxic in the Ames and DNA repair tests. Negative in vitro results for mutagenicity studies in mouse lymphoma cells and the in vivo micronucleus test have been reported.?

\section{Drug formulation}

Safinamide is water-soluble and has been formulated as 5 , 10,50 , and $200 \mathrm{mg}$ oral tablets for use in clinical trials.

\section{Drug interactions}

In vitro studies show that safinamide has no inhibiting activity on various cytochrome $\mathrm{P} 450$ isoenzymes (CYP2C9, CYP2C19, CYP2D6, CYP2E1, CYP3A4) involved in the metabolism of drugs, except CYP1A1/2, but this is at a negligible level at therapeutic doses. ${ }^{22}$

Enzyme-inducing antiepileptic drugs (eg, phenobarbital and carbamazepine) decrease plasma concentration of safinamide by approximately $30 \%$ and shorten the half-life of safinamide, but safinamide does not affect plasma concentrations of carbamazepine, phenobarbital, valproic acid, or lamotrigine. ${ }^{7}$

In one open-label, single-dose, placebo-controlled trial there was no synergistic action of safinamide with tyramine in raising blood pressure; similar results were reported in another study. ${ }^{26,27}$

\section{Clinical trials}

In an open-label pilot study, doses of safinamide (100, 150, and $200 \mathrm{mg})$ administered along with a DA $(\mathrm{n}=13)$ led to an improvement in motor performance over a 6-week period, with a significant improvement in the Unified Parkinson's Disease Rating Scale (UPDRS) motor score (measured by part III of the UPDRS) (4.2 points, $P<0.001$ ). In association with levodopa, the same doses of safinamide in eleven patients induced a significant decrease in motor fluctuations (UPDRS part IV, 2.1 points, $P<0.001$ ), accompanied by a dose-proportional increase of the area under the plasma concentration-time curve for levodopa, ranging from $56 \%$ at the dose of $100 \mathrm{mg} /$ day to $88 \%$ at the dose of $200 \mathrm{mg} /$ day. $^{28}$

In another randomized, placebo-controlled trial involving 168 patients, Stocchi et $\mathrm{al}^{29}$ reported that a median safinamide dose of $70 \mathrm{mg} /$ day (range $40-90 \mathrm{mg} /$ day) significantly increased the percentage of parkinsonian patients improving their UPDRS motor scores by a third or more from baseline (labeled as "responders") after 3 months from 21.4\% (placebo) to $37.5 \%(P<0.05)$. In a subgroup of 101 patients under stable treatment with a single DA, addition of safinamide significantly magnified the response compared with placebo (47.1\% responders, mean UPDRS motor score [part III] improvement of 4.7 points, $P=0.016$ ). No significant differences for adverse events were noted between safinamide and the placebo. ${ }^{29}$

Stocchi et al ${ }^{30}$ reported the findings of a second 24 -week, randomized, double-blind study. Patients with early PD receiving a stable dose of a single DA were randomized to once-daily safinamide $100 \mathrm{mg}(\mathrm{n}=90), 200 \mathrm{mg}(\mathrm{n}=89)$, or a placebo $(n=90)$. Mean improvement from baseline to week 24 in the primary endpoint, the UPDRS motor score (part III), was significant for safinamide $100 \mathrm{mg}$ (6.0 points difference; $P=0.0419$, versus placebo) but not for $200 \mathrm{mg}$ (3.9 points difference; $P=0.6504$, versus placebo). No clinically significant differences between safinamide and the placebo were observed for any safety variables. The most common adverse events were nausea, headache, upper abdominal pain, vomiting, pyrexia, cough, hypertension, blurred vision, gastritis, peripheral edema, nasopharyngitis, dizziness, back pain, and tremor. The incidence of these adverse events was less than $10 \%$ in each group; however, $21.3 \%$ of patients discontinued the safinamide $200 \mathrm{mg}$ dose, compared with $10.0 \%$ for the safinamide $100 \mathrm{~g}$ dose and $10.0 \%$ for the placebo. ${ }^{30}$

Anand et a ${ }^{31}$ reported two Phase III studies in PD patients with motor fluctuations using safinamide as add-on therapy. In the first study, involving 669 patients, treatment was safinamide $50 \mathrm{mg}$ /day $(\mathrm{n}=223), 100 \mathrm{mg} /$ day $(\mathrm{n}=224)$, or a placebo $(n=222)$. The primary endpoint was "on" time with no or minor dyskinesia. After 6 months, "on" time was significantly increased by 0.6 hours in both treatment groups compared with the placebo group. From the total of 669 patients in this study, 544 patients were then followed for another 18 months, with a primary endpoint of change from baseline to month 24 in dyskinesia rating scale score during "on" time. There was an improvement at 2 years in the dyskinesia rating scale score during "on" time, but this 
was not significant (50 mg/day, $P=0.21$, versus placebo; $100 \mathrm{mg} /$ day, $P=0.15$, versus placebo). The significant improvement in "on" time was maintained at month 24 : safinamide $50 \mathrm{mg}$ /day increased the average daily "on" time with no or minor dyskinesia by 0.67 hours, while $100 \mathrm{mg}$ /day gave an increase of 0.83 hours. The three most common side effects were dyskinesia, dry mouth, and back pain. ${ }^{31,32}$

\section{Other drugs in the same class}

Lazabemide, another reversible MAO-B inhibitor, showed symptomatic effects similar to those of selegiline in a doubleblind, placebo-controlled trial but was withdrawn by the study sponsor. ${ }^{33-35}$

\section{Conclusion}

Safinamide has been evaluated as an add-on to DA therapy in the early stages of PD, as an adjunct to levodopa in the middle stages of PD, and as an antidyskinetic agent, showing some efficacy on all counts. Safinamide is comparable with the two other MAO-B inhibitors licensed for treatment of $\mathrm{PD}$, selegiline and rasagiline, in that it does not have dietary restrictions for food with high tyramine content and can be used once daily because of its relatively long halflife. It has been studied in a large number of PD patients at doses ranging between 50 and $200 \mathrm{mg}$ /day without serious side effects. There have been no direct comparisons made with selegiline or rasagiline. Two further Phase III trials, MOTION (safinamide add-on to DAs for early idiopathic PD) and SETTLE (safinamide in idiopathic PD with motor fluctuations, as add-on to levodopa), have yet to report. Merck $\mathrm{KGaA}$ recently returned the rights to develop, manufacture, and commercialize safinamide for PD to Newron Pharmaceuticals $\mathrm{SpA}$.

\section{Future directions}

Drugs that are the subject of current trials for treatment of PD include modified formulations of levodopa (IPX066), DAs (pardoprunox), COMT inhibitors (nebicapone), adenosine receptor antagonists (istradefylline, preladenant), neurotrophic factors $\left(\operatorname{Cogane}^{\circledR}\right)$, gene therapies utilizing an adeno-associated virus vector to deliver the glutamic acid decarboxylase gene, stem cell therapies, antidyskinetic agents (fipamezole), and drugs for treatment of excessive daytime sleepiness (pitolisant). Nondopaminergic mechanisms play a role in many of the nonmotor manifestations of PD and are therefore a key current focus of research. Dopaminergic therapies underpin current management strategies in treating PD, and their evolution continues with agents such as safinamide. It is also anticipated that the scope of antiparkinsonian therapy will broaden, with new treatments that influence nondopaminergic mechanisms.

\section{Acknowledgments/disclosure}

DG has received consultancy fees from Civitas Therapeutics Inc and Vectura Group plc and honoraria from Teva/Lundbeck, UCB Pharma, GlaxoSmithKline, Pharmacia, and Abbott. NM reports no conflicts of interest in this work.

\section{References}

1. Hughes AJ, Daniel SE, Kilford L, Lees AJ. Accuracy of clinical diagnosis of idiopathic Parkinson's disease: a clinico-pathological study of 100 cases. J Neurol Neurosurg Psychiatry. 1992;55(3): 181-184.

2. BirkmayerW, Hornykiewicz O. The effect of 1-3,4-dihydroxyphenylalanine (=DOPA) on akinesia in parkinsonism. Parkinsonism Relat Disord. 1998;4(2):59-60.

3. Kitamura Y, Taniguchi T, Shimohama S, Akaike A, Nomura Y. Neuroprotective mechanisms of antiparkinsonian dopamine D2-receptor subfamily agonists. Neurochem Res. 2003;28(7):1035-1040.

4. Gray R, Patel S, Ives N, et al; for PD MED Collaborators. A large randomised trial assessing the quality of life in patients with early PD: results from PD MED EARLY [platform and poster presentation]. XIX World Congress on Parkinson's Disease and Related Disorders; December 11-14, 2011; Shanghai, People's Republic of China.

5. Ilani T, Lamensdorf I, Finberg JP. Selective monoamine oxidase subtype inhibition and striatal extracellular dopamine in the guinea-pig. $\mathrm{Br} \mathrm{J}$ Pharmacol. 2000;130(8):1992-1998.

6. Fariello RG. Safinamide. Neurotherapeutics. 2007;4(1):110-116.

7. Bialer M, Johannessen SI, Kupferberg HJ, Levy RH, Perucca E, Tomson T. Progress report on new antiepileptic drugs: a summary of the Seventh Eilat Conference (EILAT VII). Epilepsy Res. 2004;61(1-3):1-48.

8. Fariello RG, McArthur RA, Bonsignori A, et al. Preclinical evaluation of PNU-151774E as a novel anticonvulsant. J Pharmacol Exp Ther. 1998;285(2):397-403.

9. Maj R, Fariello RG, Ukmar G, et al. PNU-151774E protects against kainate-induced status epilepticus and hippocampal lesions in the rat. Eur J Pharmacol. 1998;359(1):27-32.

10. Salvati P, Maj R, Caccia C, et al. Biochemical and electrophysiological studies on the mechanism of action of PNU-151774E a novel antiepileptic compound. J Pharmacol Exp Ther. 1999;288(3):1151-1159.

11. Matos M, Viña D, Quezada E, et al. A new series of 3-phenylcoumarins as potent and selective MAO-B inhibitors. Bioorg Med Chem Lett. 2009; 19(12):3268-3270.

12. Binda C, Hubálek F, Li M, et al. Crystal structures of monoamine oxidase $\mathrm{B}$ in complex with four inhibitors of the $\mathrm{N}$-propargylaminoindan class. J Med Chem. 2004;47(7):1767-1774.

13. Fernandez HH, Chen JJ. Monoamine oxidase-B inhibition in the treatment of Parkinson's disease. Pharmacotherapy. 2007; 27(12 Pt 2):S174-S185.

14. Caccia C, Maj R, Calabresi M, et al. Safinamide: from molecular targets to a new anti-parkinson drug. Neurology. 2006;67(7 Suppl 2): S18-S23.

15. Sawada H, Oeda T, Kuno S, et al; for Amantadine Study Group. Amantadine for dyskinesias in Parkinson's disease: a randomized controlled trial. PLoS One. 2010;5(12):e15298.

16. Metman LV, Del Dotto P, LePoole K, Konitsiotis S, Fang J, Chase TN. Amantadine for levodopa-induced dyskinesias: a 1-year follow-up study. Arch Neurol. 1999;56(11):1383-1386.

17. Pevarello $\mathrm{P}$, Bonsignori A, Caccia C, et al. Sodium channel activity and sigma binding of 2-aminopropanamide anticonvulsants. Bioorg Med Chem Lett. 1999;9(17):2521-2524. 
18. Binda C, Wang J, Pisani L, et al. Structures of human monoamine oxidase B complexes with selective noncovalent inhibitors: safinamide and coumarin analogs. J Med Chem. 2007;50(23):5848-5852.

19. Tsai SY, Rothman RK, Su TP. Insights into the sigma-1 receptor chaperone's cellular functions: a microarray report. Synapse. 2012; 66(1):42-51.

20. Mishina M, Ishiwata $\mathrm{K}$, Ishii $\mathrm{K}$, et al. Function of sigma1 receptors in Parkinson's disease. Acta Neurol Scand. 2005;112(2):103-107.

21. Bonuccelli U, Del Dotto P. New pharmacologic horizons in the treatment of Parkinson disease. Neurology. 2006;67(7 Suppl 2):S30-S38.

22. Marzo A, Dal Bo L, Monti NC, et al. Pharmacokinetics and pharmacodynamics of safinamide, a neuroprotectant with antiparkinsonian and anticonvulsant activity. Pharmacol Res. 2004;50(1):77-85.

23. Luszczki JJ. Third-generation antiepileptic drugs: mechanisms of action, pharmacokinetics and interactions. Pharmacol Rep. 2009;61(2): 197-216.

24. Vaghi F, Maj R, Rosa B, et al. Neuroprotective effect of PNU-151774E, a new anticonvulsant compound, in the model of global ischaemia in gerbils. Soc Neurosci Abstr. 1997;23:545.

25. Kupsch A, Sautter J, Götz ME, et al. Monoamine oxidase-inhibition and MPTP-induced neurotoxicity in the non-human primate: comparison of rasagiline (TVP 1012) with selegiline. J Neural Transm. 2001;108(8-9):985-1009.

26. Cattaneo C, Caccia C, Marzo A, Maj R, Fariello RG. Pressor response to intravenous tyramine in healthy subjects after safinamide, a novel neuroprotectant with selective, reversible monoamine oxidase B inhibition. Clin Neuropharmacol. 2003;26(4):213-217.

27. Di Stefano AF, Rusca A. Pressor response to oral tyramine during co-administration with safinamide in healthy volunteers. Naunyn Schmiedebergs Arch Pharmacol. 2011;384(6):505-515.
28. Stocchi F, Vacca L, Grassini P, et al. Symptom relief in Parkinson disease by safinamide: biochemical and clinical evidence of efficacy beyond MAO-B inhibition. Neurology. 2006;67(7 Suppl 2):S24-S29.

29. Stocchi F, Arnold G, Onofrj M, et al; for Safinamide Parkinson's Study Group. Improvement of motor function in early Parkinson disease by safinamide. Neurology. 2004;63(4):746-748.

30. Stocchi F, Borgohain R, Onofrj M, et al; for Study 015 Investigators. A randomized, double-blind, placebo-controlled trial of safinamide as add-on therapy in early Parkinson's disease patients. Mov Disord. 2012;27(1):106-112.

31. Anand R, Borgohain R, Stocchi F, et al; for Study 016/018 Investigators. First 2-year, placebo-controlled study in Parkinson's disease patients with motor fluctuations indicates safinamide may benefit patients with more severe dyskinesia. Parkinsonism Relat Disord. 2012;18 Suppl 2: S132-S133.

32. Szasz J, Borgohain R, Bhatt M, Rossetti S, Lucini V, Anand R; for Study 016 Investigators. Effects of safinamide as add-on to L-dopa on symptom severity and aspects of daily living in patients with mid- to late-stage Parkinson's disease. 2nd World Parkinson Conference; 2010 Sep 28-Oct 1; Glasgow, Scotland.

33. Parkinson Study Group. A controlled trial of lazabemide (Ro 19-6327) in levodopa-treated Parkinson's disease. Arch Neurol. 1994;51(4): 342-347.

34. LeWitt PA, Segel SA, Mistura KL, Schork MA. Symptomatic antiparkinsonian effects of monoamine oxidase-B inhibition: comparison of selegiline and lazabemide. Clin Neuropharmacol. 1993;16(4): 332-337.

35. Parkinson Study Group. Effect of lazabemide on the progression of disability in early Parkinson's disease. Ann Neurol. 1996;40(1): 99-107.
Journal of Experimental Pharmacology

\section{Publish your work in this journal}

The Journal of Experimental Pharmacology is an international, peerreviewed, open access journal publishing original research, reports, reviews and commentaries on all areas of laboratory and experimental pharmacology. The manuscript management system is completely online and includes a very quick and fair peer-review system.

\section{Dovepress}

Visit http://www.dovepress.com/testimonials.php to read real quotes from published authors. 